\title{
La science, les politiques et le public : quelle réalité, quels écueils?
}

\author{
Sander van der Leeuw \\ Professeur, ancien doyen de la School of Sustainability, Arizona State University, Tempe, États-Unis
}

\begin{abstract}
Mots-clés :
illettrisme

scientifique ;

perte de confiance

dans les sciences ;

fragmentation

disciplinaire ; langage

«STEM » (sciences,

technologie,

ingénierie,

Résumé - Il s'agit ici de regarder les sciences dans une perspective ethnographique, en soulignant que la création des résultats scientifiques et leur évaluation par le public sont sociales et s'inscrivent dans des processus contextuels. La perte de la crédibilité scientifique au sein d'un large public, aux États-Unis en particulier, semble due au fait qu'après la Seconde Guerre mondiale, les sciences ont créé un niveau d'attente irréaliste et ont eu des conséquences indésirables et inattendues, alors que la pratique scientifique devenait de plus en plus liée à la sphère commerciale et aux gouvernements. Dans ce contexte, l'effondrement relatif de l'éducation en matière de science, de technologie, d'ingénierie et de mathématiques (STEM) fut, aux États-Unis, particulièrement dommageable. Recouvrer la confiance d'une large part de la société implique de reconsidérer les pratiques et la communication scientifiques, ainsi que le contexte institutionnel dans lequel les sciences sont mises en œuvre.
\end{abstract}

mathématiques)

\section{Keywords:}

scientific illiteracy;

loss of trust in science; disciplinary

fragmentation;

STEM language

\begin{abstract}
Science, politics and the general public: what is the reality, what are the pitfalls? This paper looks at the sciences from an ethnographic perspective, emphasizing that both the creation of scientific results and their evaluation among the general public are social and contextual processes. The loss of scientific credibility among the wider public, particularly in the US, seems due to the fact that after WWII the sciences raised unrealistic expectations and had many unintended and undesirable consequences, while the practice of science became more and more entwined with both business and government. In that context, the relative decay of education in the language of science, technology, engineering and mathematics (STEM) in the US is of particular importance. To regain the trust of the wider society, a reconsideration of the ways in which we practice and communicate science, as well as the institutional context in which the sciences are practiced, is called for.
\end{abstract}

\section{Introduction : une perspective anthropologique des rapports science et société}

Dans cet article ${ }^{1}$, je me propose de regarder d'un peu plus près les difficultés de communication rencontrées lors des discussions concernant le développement durable et le changement climatique - plus particulièrement entre les scientifiques, les «stakeholders » et le grand public. Ces difficultés ont généré une réticence à admettre certaines des conclusions de la communauté scientifique et sont l'une des nombreuses causes du retard dans la mise en œuvre des mesures constructives de lutte contre les changements environnementaux. Cela est particulièrement évident pour ce qui concerne les États-Unis.

Dans la communauté scientifique, ces problèmes de compréhension sont souvent considérées comme le

Auteur correspondant : vanderle@asu.edu

1 Ce texte est une version écrite d'une communication faite le 28 août 2011 à l'Université européenne d'été de l'Institut des hautes études pour la science et la technologie, intitulée «Science, culture, éducation : des sociétés guettées par l'illettrisme scientifique?». 
résultat d'un illettrisme scientifique, à savoir une incapacité présumée des décideurs et du grand public à comprendre les sciences et l'importance de leurs conclusions.

Mais dans la perspective de l'anthropologue, ces difficultés ne sont pas différentes de celles qui émergent lorsque plusieurs cultures interagissent. Tout un chacun qui a voyagé ou habité dans une société autre que la sienne est évidemment conscient que, "chez autrui », on pense et on agit de manière différente que «chez soi ». L'anthropologie est l'étude de l'autre afin de comprendre, plutôt que de juger. Il s'agit explicitement d'une vision « neutre » qui n'accorde aucune valeur - positive ou négative - aux cultures, afin de pouvoir les comparer et en étudier les origines et les dynamiques de manière aussi « objective » que possible.

Je considère la science comme un ensemble d'idées acquises et portées par une communauté sociale (les chercheurs et tous ceux qui acceptent leurs méthodes, raisonnements et résultats), comme une « culture » scientifique acquise par apprentissage dans un contexte social, au même titre qu'une culture « religieuse », « chinoise » ou « anglo-saxonne ». Notre vision est donc à la fois une vision anthropologique de la culture scientifique de tradition « occidentale » et une vision tout aussi anthropologique du contexte culturel et social dans lequel notre communauté scientifique est imbriquée.

\section{L'illettrisme scientifique et ses racines}

Ce qu'on appelle - à tort - l'illettrisme scientifique est un phénomène important dans les pays occidentaux. Il n'est nulle part aussi répandu qu'aux États-Unis, où presque un tiers de la population - environ 100 millions de personnes - ferme délibérément ses oreilles (et son cerveau) lorsqu'on parle de science, et plus particulièrement des avertissements des scientifiques à propos des changements climatiques, parce qu'elle ne veut pas y croire.

D'un point de vue anthropologique - et contrairement à ce que peut parfois en penser une partie du corps académique - il ne s'agit pas d'une mauvaise volonté, d'une absence de compréhension, d'un manque d'intelligence ou de connaissance scientifique. Il s'agit plutôt d'une mise en œuvre différente - non scientifique - pour rendre le monde compréhensible et acceptable. Une fois acquise et intégrée, cette perspective est tellement distante de la perspective scientifique qu'il devient très difficile, voire impossible, de les combiner.

Le rapport entre la «culture scientifique » et la « culture non scientifique " n'est donc pas - dans notre vision - fondamentalement différent de ce qui distingue deux cultures, française et anglo-saxonne, par exemple, ou deux cultures disciplinaires académiques comme celles des sciences naturelles et des SHS, qui ont des difficultés à communiquer. Chacune a sa propre manière de voir les choses, ses propres valeurs et son propre langage que $\mathrm{l}^{\prime}$ on doit respecter lorsqu'on travaille ensemble.

À l'origine de telles différences "culturelles », on trouve des différences dans l'intensité de communication intra- et intercommunautés. Sur la longue durée, toute communauté dont les membres communiquent de manière intense et fréquente développe des mots, des concepts - et même une langue et une identité - qui lui sont propres, et qui sont de moins en moins compréhensibles pour les non-membres (ceux qui interagissent peu avec elle) parce qu'ils constituent une autre communauté. Dans notre cas, la culture, le langage, les institutions et les pratiques de la communauté scientifique se sont donc différenciées pendant plusieurs siècles de la culture, du langage, des institutions et des pratiques non scientifiques, jusqu'au point où il est devenu difficile pour les non-initiés de comprendre cette communauté scientifique, et vice versa. Je présente plus loin quelquesunes de ces différences qui ne sont pas évidentes au premier regard. Mais avant de ce faire, il me semble utile de souligner deux caractéristiques de chacune de ces perspectives.

Tout d'abord, chaque perspective est réductrice. Toutes nos expériences du monde extérieur passent par un filtre cognitif qui en réduit très fortement la complexité perçue et retenue ; car le nombre de dimensions connues et manipulables conjointement est limité par la capacité de notre "mémoire de travail » (STWM : Short Term Working Memory) (Read et van der Leeuw, 2009; van der Leeuw, 2010). Ainsi nous retenons, par exemple, les caractéristiques d'un arbre qui nous frappent le plus, mais nous ne retenons pas l'infinité des autres. Il s'ensuit que nos interventions dans l'environnement sont basées sur une vision réductrice de ce qu'il s'y passe réellement, mais qu'elles affectent toutes ses dimensions inaperçues. Ainsi, chaque intervention humaine dans l'environnement aboutit à un nombre important (mais inconnu) de «conséquences inattendues» qui ne se manifestent qu'ultérieurement.

Qui plus est, nos idées et théories sont sous-déterminées par nos observations (Atlan, 1992). Car nous n'en disposons jamais en nombre suffisant pour pouvoir évaluer toutes les (très nombreuses) théories qui pourraient en principe expliquer le comportement d'un système complexe de manière concluante. En conséquence, la grande majorité de nos idées et théories est en effet surdéterminée par nos expériences antérieures. C'est en nous appuyant sur ces expériences antérieures que nous sélectionnons les caractéristiques de chaque phénomène, chaque processus. Comme on le dit en anglais, l'évolution d'une vision du monde, d'une discipline, 
d'une culture est donc path dependent - elle dépend de son histoire. Et cela au niveau individuel comme au niveau du groupe!

La science - comme toute autre façon d'expliquer le monde environnant $-\mathrm{s}$ 'acquiert chez les enfants dès leur plus jeune âge, dans le contexte familial, scolaire et social. Or, aux États-Unis, les enfants ne sont ni obligés d'aller à l'école ni de suivre un cursus commun. Qui plus est, l'investissement dans le système éducatif se décide au niveau local et apparait, au regard des normes européennes, souvent très en deçà du nécessaire. Beaucoup d'écoles sont gérées par des structures religieuses ou sociales qui établissent un cursus ad hoc, afin de promouvoir la spécificité de leurs idées. Il n'est donc guère étonnant qu'une partie importante de la population nordaméricaine ne souscrive pas à l'approche scientifique.

La situation en Europe est différente. La scolarité y est obligatoire dans beaucoup de pays ainsi que des enseignements scientifiques. Les scores aux tests conventionnels d'évaluation des connaissances scientifiques y sont donc souvent plus élevés qu'aux États-Unis. Mais, comme en témoigne, par exemple, l'opposition aux OGM, la familiarité des Européens avec les sciences les mène à un certain scepticisme envers certaines conclusions, plutôt qu'à une adhésion sans réserve à la méthode scientifique. Par ailleurs, certains comportements démontrés comme dangereux - comme le tabagisme - sont plus facilement acceptés en Europe ; cela implique que la présence ou l'absence de connaissances scientifiques n'est donc pas le seul facteur qui détermine les attitudes d'une population.

\section{Crises et rôle des sciences au sein de notre société}

Notre société est aujourd'hui confrontée à la conjonction de plusieurs crises: sociale, écologique, économique, financière, etc. Or, en l'absence d'un flux suffisant d'informations entre ses membres, c'est-à-dire sans une interaction suffisamment fréquente entre eux, toute société est condamnée à s'effondrer. Maintenir un tel flux, qui permet à une société de s'organiser et de répondre de manière cohérente aux défis qu'elle rencontre, exige une large et profonde participation de la population sur le plan tant de ses représentations culturelles, que de son langage, de ses institutions, de ses mœurs et de ses coutumes. Sans cette participation, une société génère trop de malentendus, voire trop de conflits qui, après un certain temps, conduisent à une perte de sa cohérence dès que des intérêts individuels ou de certains groupes sociaux priment sur ceux de la société dans son ensemble.

Un problème de fond qui sous-tend la crise socioéconomique et environnementale actuelle est l'absence d'une culture et d'une vision du monde suffisamment partagées par l'ensemble des acteurs sociaux impliqués, car seule une communauté de vues permettrait de gérer cette période dans toute la pluralité de ses formes. On pourrait définir l'actuelle situation comme « une incapacité temporaire de la société à traiter conjointement les différentes informations nécessaires à la bonne gestion des dynamiques auxquelles elle a affaire ». Du fait de cette inaptitude, nous n'abordons à chaque fois qu'un ou deux aspects de la situation actuelle parmi la multitude de ceux qui la constituent.

En outre, l'incapacité politique à résoudre les crises financières récurrentes du dollar et de l'euro montre clairement qu'il n'y a évidemment jamais une seule perception; même une représentation idéalisée et largement partagée par la société - comme dans le cas d'une guerre ou d'une catastrophe naturelle - reste toujours partielle.

Ce manque d'harmonisation politique me semble en partie dû à la dégradation - dans nos sociétés occidentales - de l'outil de communication jusqu'ici accepté par les classes dirigeantes et moyennes. La « rationalité » occidentale des interactions sociales et culturelles repose sur le langage des sciences, de la technologie, de l'ingénierie et des mathématiques (en anglais STEM : Science, Technology, Engineering, and Mathematics) qui inclut toutes les disciplines scientifiques. C'est ce langage universaliste qui a permis d'établir une conception du monde dont les bénéfices matériels sont - depuis 250 ans - indéniables. Notre capacité à surmonter la crise actuelle est donc très étroitement liée à la proportion de la population capable de communiquer dans le langage des STEM. Or, comme nous le verrons ci-dessous, l'augmentation de l'illettrisme scientifique est un signe que ce moyen de communication ne fonctionne plus aussi bien qu'au XXe siècle. Quelles pourraient être les causes de ce déclin?

\section{Les rôles respectifs de la science et de la société ont changé}

L'activité scientifique est un processus structuré par des questionnements, des observations et une organisation des savoirs. Ce processus est mis en œuvre et développé par une communauté sociale de chercheurs, d'ingénieurs et de techniciens de différentes disciplines. La société dont ils font partie détermine le contexte dans lequel ce processus se déroule. Elle participe à la définition des questions posées et aux moyens d'observation. Elle influe également sur les valeurs à l'aune desquels les résultats sont interprétés. En réalité, des « faits » acquis de manière « objective » répondent à des questions autoréférencées et négociées de manière subjective. Dans cette perspective, la pratique scientifique n'est donc pas de décider entre « le vrai » ou « le faux », mais de définir 


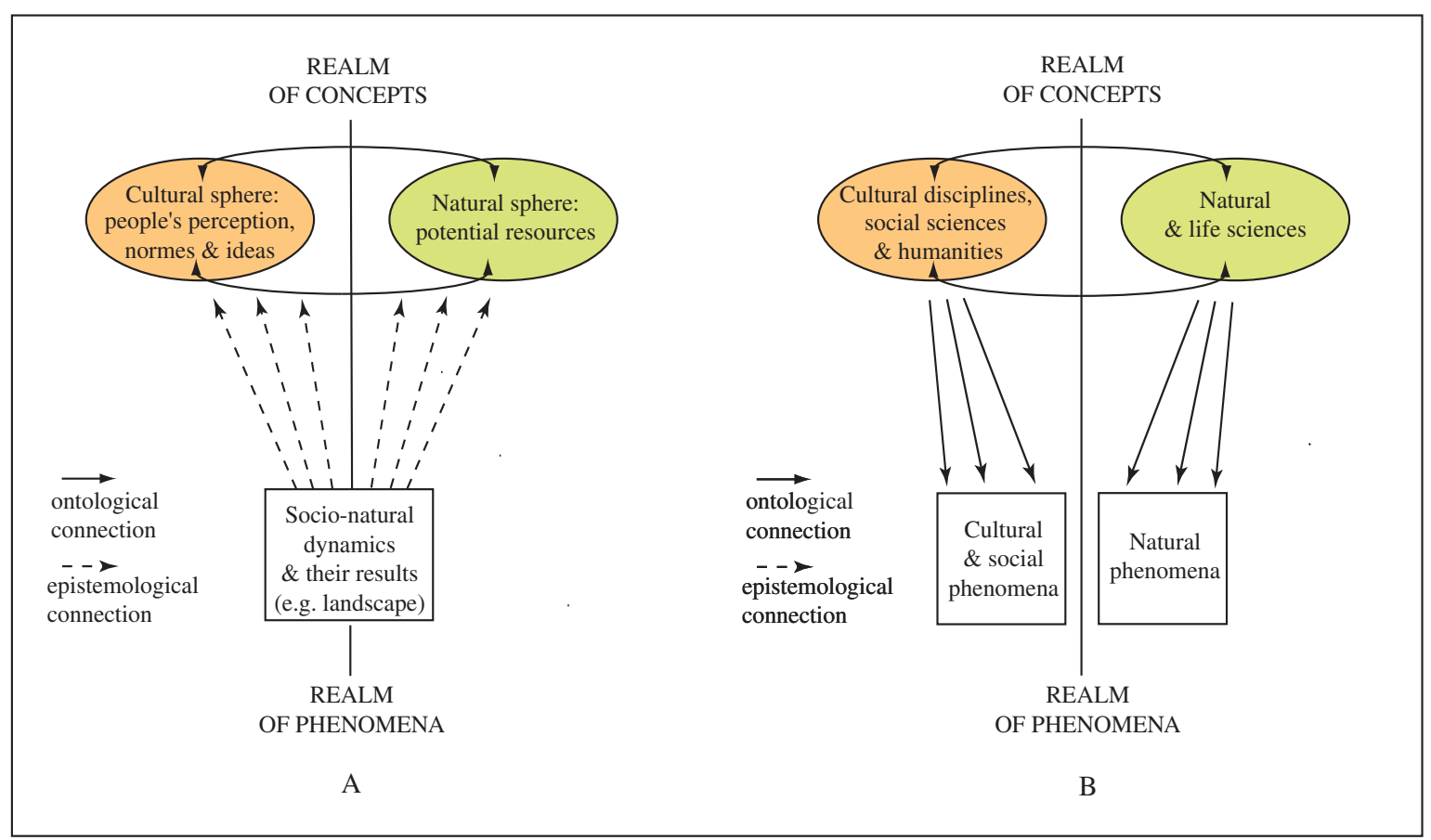

Fig. 1. Liens entre concepts et phénomènes. L'émergence des disciplines a inversé la logique des sciences. Initialement (à gauche sur la figure), le lien entre le domaine des phénomènes et celui des concepts était un lien épistémologique, mais quand les méthodes et les techniques ont constitué le socle des disciplines, ces liens sont devenus ontologiques (à droite sur la figure) ; progressivement, les méthodes et les techniques acquises ont commencé à prédominer dans le choix des questions et des défis à investiguer. Cela a stimulé une spécialisation de plus en plus étroite, et a conduit à des difficultés de communication entre communautés disciplinaires (source : Sander van der Leeuw, 2011).

et de déterminer le degré de validité des observations dans un contexte social donné. La connaissance ainsi produite sert à construire cette société ; elle est généralement fiable dans ce contexte, dans lequel elle est profondément ancrée et intégrée.

Depuis deux siècles environ, les sciences ont progressivement été institutionnalisées, ce qui les a modifiées en profondeur. Il y a deux siècles et demi, des chercheurs ("amateurs savants") étudiaient des phénomènes de toutes sortes, et se posaient des questions a priori sans limites (Fig. 1A). Selon leurs expériences, ils appliquaient des méthodes variées qu'ils avaient soit déjà utilisées, soit inventées. Il n'y avait pas de démarche systématique d'étude ou d'analyse. Pour simplifier, on pourrait dire qu'en fonction de leurs observations dans le monde "réel ", ils classaient les phénomènes étudiés dans diverses catégories conceptuelles, par exemple, "phénomènes naturels » ou "phénomènes sociaux ».

Avec l'institutionnalisation et la formalisation du monde académique et de la recherche, depuis la moitié du XIX ${ }^{\mathrm{e}}$ siècle, les relations entre observations et concepts se sont, petit à petit, inversées. La structuration disciplinaire de la recherche et de la formation qui en a résulté a limité le champ des enquêtes de chacun à sa discipline. Progressivement, ce sont les idées acquises dans la formation disciplinaire qui ont déterminé les questions posées, les phénomènes étudiés et les méthodes d'investigation utilisées (Fig. 1B). Ce sont nos théories (disciplinaires) qui déterminent désormais la direction d'évolution de nos connaissances, et non plus nos observations.

Ainsi, les relations entre phénomènes et concepts (idées, théories, questions), qui étaient à l'origine épistémologiques, sont devenues ontologiques, et notre vision du monde s'est fragmentée en perspectives disciplinaires et sectorielles (par exemple, celles des «sciences naturelles » et des "sciences sociales », ou encore celles de la biologie moléculaire et de la tectonique des plaques) qui rendent une vision globale, transdisciplinaire ou holiste difficile à développer.

Dans le même temps, les relations sciences-société ont aussi changé : les sciences sont désormais reconnues comme source d'innovation pour l'industrie (et donc source de valeur pour l'économie), et, dans le domaine politique, beaucoup d'institutions, de processus et de décisions invoquent la science comme justification. Elle est donc devenue un enjeu sociétal d'importance.

Or, le public ne perçoit jamais les STEM de manière purement intellectuelle. Les activités et les opinions scientifiques sont toujours ressenties et appréciées en tant qu'expressions de relations matérielles, sociales et politiques, d'interactions et d'intérêts. Le principal facteur qui gouverne le rôle et la perception des sciences est 
la confiance que le public est prêt à investir dans les institutions scientifiques et les individus qui les représentent. Et cette confiance est soumise aux aléas des opinions qui circulent dans les réseaux sociaux. Au fur et à mesure que la société a été déçue dans ses attentes des STEM, elle a perdu sa confiance dans les sciences et s'est ouverte à d'autres idées, conduisant, entre autres, à ce que le monde scientifique appelle "l'illettrisme scientifique ».

Dans nos sociétés occidentales, les STEM ne répondent en effet plus de manière efficace à nos attentes pour deux raisons principales. D'une part, ces attentes ne sont plus très réalistes, et sont de plus en plus déçues, après un siècle de succès scientifiques quasiment ininterrompus. Il est en effet devenu de plus en plus difficile $\mathrm{d}$ 'introduire des innovations radicalement nouvelles, comme le montre une analyse des brevets octroyés par les autorités américaines depuis 1789 (Strumsky et al., 2011). D'autre part, de multiples succès de la « technologie " occidentale ont eu aussi des "conséquences inattendues » que nous observons dans notre environnement. Leur nombre augmente rapidement, et elles brouillent ainsi notre vision du futur. Nous sommes, pour l'heure, confrontés à ce que Daniel Kahnemann et Amos Tversky (1979) appellent «the planner's fallacy », à savoir l'illusion de croire que notre tendance à toujours innover pourrait nous conduire à un futur totalement intentionnel et contrôlé !

En revanche, cette confiance existe et se déploie dans les BRICS (Brésil, Russie, Inde, Chine, Afrique du Sud) et autres pays en voie de développement rapide. À ce sujet, il faudrait peut-être considérer que ces différences pourraient être liées au fait que les BRICS se focalisent sur les conséquences attendues des STEM pouvant stimuler leur activité économique, tandis que les pays «occidentaux » sont actuellement confrontés aux conséquences inattendues de leur " développement », ce qui rend leurs progrès plus difficiles.

\section{L'exemple de Sellafield}

Afin de mieux comprendre l'articulation entre dynamiques sociétales et dynamiques scientifiques, il convient d'analyser quelques-unes de leurs différences fondamentales. Pour ce faire, reportons-nous d'abord à l'étude bien connue de Brian Wynne sur la perception de l'impact de Tchernobyl sur l'environnement des éleveurs de moutons dans le nord de l'Angleterre, près de la centrale nucléaire (très controversée) de Sellafield (Wynne, 1996).

Après l'accident de Tchernobyl, les scientifiques britanniques - chargés de mesurer la radioactivité locale afin de déterminer les dangers et de proposer d'éventuelles mesures pour protéger la population et ses activités économiques - ont fait deux grandes erreurs. Tout d'abord, en raison de leur grande confiance dans leur métier et leur expertise personnelle, leur langage n'a pas pris en compte l'imprévisibilité et les imprévus que la population avait l'habitude de gérer au quotidien. En outre, les conseils de ces chercheurs se sont basés sur un modèle des effets du césium sur les sols qui concernait des sols argileux, et qui n'était pas approprié pour la région de Sellafield. Notamment, alors que l'argile immobilise le césium, celui-ci reste mobile dans les sols tourbeux de la région concernée. Ainsi, les avis des scientifiques, fondés sur leurs prédictions de l'intensité de la radioactivité du sol, étaient beaucoup plus alarmants que les observations de la population locale, faites par eux au dosimètre. La confiance des éleveurs dans les recommandations officielles s'en est trouvée fortement réduite. Ce qui a conduit à une crise de légitimité, dans laquelle les protagonistes ont justifié leurs idées en fonction de leur identité sociale, plutôt qu'en fonction des réalités du terrain et de l'applicabilité de ces idées.

Une fois la confiance des éleveurs dans les chercheurs envolée, les premiers ont jugé que les deuxièmes constituaient un danger pour la cohérence de leur société locale. Dans ce cas précis, ils ont estimé que les chercheurs - envoyés par le gouvernement - tentaient de cacher les vrais risques de la centrale nucléaire de Sellafield, et non de les aider à surmonter les dangers du nuage radioactif de Tchernobyl.

\section{Leçons à tirer de l'exemple ci-dessus}

Une première leçon à tirer de cet épisode concerne les différences entre la prospective scientifique et la vision du futur par les acteurs sociaux. L'expérience ex ante des agriculteurs/bergers est celle du doute, de l'imprévisibilité et des risques..., une expérience acquise tout au long de leur vie, qui les a toujours amenés à gérer les conséquences inattendues de leurs actions face aux nombreux paramètres imprévisibles (météo, marchés, santé des animaux, etc.). Mais la perspective scientifique ex ante est, quant à elle, soit celle de la certitude, soit celle de l'incertitude, ce qui limite intellectuellement la valeur prédictive de la science. Les chercheurs acceptent l'incertitude comme une limitation à ce qu'ils peuvent dire, mais ils n'en dépendent pas dans leur carrière ou leur vie quotidienne. Ils ne ressentent donc pas le besoin d'y répondre, et n'ont pas trouvé la manière constructive d'en discuter avec des personnes quotidiennement confrontées à l'incertitude.

Dans ce contexte, il est également intéressant d'analyser le rôle de l'ambiguïté. Les sciences cherchent à supprimer les contradictions inhérentes à leurs observations, afin de pouvoir proposer des théories claires, nettes et sans ambiguiités ; ce faisant, elles construisent nécessairement une vision irréelle du monde - une 
vision simplifiée qui n'explique pas toutes les observations et qui ne prend pas en considération des questions auxquelles elles ne peuvent répondre. En revanche, les acteurs sociaux acceptent les contradictions insolubles, la complexité de la réalité et leur propre incapacité à comprendre et à contrôler cette réalité. Dans l'exemple ci-dessus, les bergers ne peuvent évidemment pas éviter d'affronter l'imprévisibilité du monde qu'ils doivent gérer. Ambivalence et ambiguïté jouent donc un rôle majeur dans leur vision du monde.

Un troisième aspect concerne les rôles respectifs des « observations » et des «théories » dans les deux communautés. La communauté scientifique cherche à construire des théories qui peuvent expliquer, du moins en partie, la réalité observée, tandis que la communauté non scientifique tâche de " gérer » cette réalité sans pour autant l'expliquer. En science, l'important est de lier étroitement théorie et observations, tandis que cette relation est moins forte hors science : on peut avoir des théories mais ce qui compte c'est la réalité. Les dimensions de cette réalité sont en nombre quasi infini ; elle est toujours plus riche et complexe que les théories la concernant, et nos observations peuvent s'interpréter de multiples manières, ce qui est moins le cas pour nos idées et nos théories en raison des limites de notre système cognitif.

Ce point s'exprime dans la différence de statut que les deux communautés accordent à leurs savoirs respectifs. Les scientifiques donnent à leurs idées, une fois acceptées par leur communauté, le statut de «savoirs » ou de " connaissances », tandis que le public donne le statut de «compréhension» ou d' $\mathrm{d}^{\prime}$ expériences » à ses idées. Dans le premier cas, le statut du « savoir » est absolu et présumé partagé, et dans le deuxième cas, il est personnel et variable.

Il s'ensuit une différence importante entre une activité «scientifique» et une activité «politique». Le domaine de la politique est ouvertement subjectif. On y gère des problèmes qui ne sont pas solubles de manière univoque ("wicked problems », selon Churchman, 1967), afin de négocier des remèdes opérationnels - pas nécessairement rationnels mais émotionnellement satisfaisants. On y cherche à prédire le futur, tout en affrontant les dimensions cachées de problèmes extrêmement complexes. En revanche, dans le domaine scientifique, on fait émerger de nombreuses " objectivités » tout en enquêtant sur les bases « irrationnelles » du domaine social, et en posant des questions pour trouver des réponses rationnelles. On cherche à expliquer le passé en réduisant le nombre des dimensions des processus et des phénomènes observés; on change également souvent de sujet et de perspective disciplinaire. Dans les deux domaines, on construit donc une perspective sousdéterminée par les observations du monde "réel », mais les deux perspectives diffèrent. La perspective scientifique est sous-déterminée selon les principes stricts, rigides et transparents des STEM, alors que la perspective politique l'est selon d'autres principes, plus émotionnels et plus flous mais tout aussi pertinents dans leur domaine.

Voici donc quelques-unes des différences fondamentales dans la façon dont les deux communautés, scientifique et non scientifique, bâtissent leur interprétation du monde et leur identité à travers celle-ci. Ce sont autant de difficultés à surmonter lorsque l'on tente de créer un pont entre la science et le grand public, en y incluant ceux que la communauté scientifique nomme actuellement « illettrés scientifiques ».

\section{Le rôle futur de la science dans nos sociétés}

Tout ce que nous venons de discuter nous mène inéluctablement à la question suivante : la confiance dans la science s'est-elle dégradée au point de compromettre son rôle crucial dans les progrès du bien-être de l'humanité ? Je ne le crois pas. Les deux derniers siècles ont permis à l'approche scientifique STEM de s'ancrer tellement profondément dans nos institutions (commerciales, financières, politiques, académiques, culturelles...), qu'une fin plus ou moins abrupte du rôle des sciences n'est pas proche. En outre, notre système cognitif, avec son penchant pour la continuité, et l'essor de l'approche scientifique dans les BRICS, plaident en faveur d'une vie encore longue des STEM.

Néanmoins, une diffusion importante de l'illettrisme scientifique pourrait avoir des conséquences importantes sur la cohésion du monde développé ; nous devons donc nous demander ce que nous pouvons faire afin de réintégrer au mieux les sciences dans notre culture.

Constatons d'abord que la communauté scientifique occidentale a présumé - à tort - qu'elle devait être un modèle de rationalisme. Mais quand divers types d'institutions gouvernantes ont adopté une perspective et une identité rationaliste, tout en cachant (ou en ignorant) les aspects politiques et "émotionnels » de leurs décisions et actions, cela a conduit à une réaction négative du grand public envers les sciences et les institutions, ce qui a mené à l'émergence de mouvements politiques extrainstitutionnels, comme le Tea Party actuel aux Etats-Unis. Il s'agit d'une « crise de la modernité tardive ».

Nous-mêmes, chercheurs, en sommes en partie responsables, puisque nous n'avons pu éviter d'être perçus comme arrogants, autocentrés, sourds et aveugles au monde qui nous entoure. Si, dans le passé, la science était perçue comme le domaine où de rares experts inventaient des choses miraculeuses, ce qui a favorisé son essor, ce temps est définitivement révolu.

Il faut admettre que le monde a changé. Nous vivons actuellement une époque où le retranchement défensif 
(dans une tour d'ivoire) n'est plus possible. Notre société, qui finance fortement ses universités, attend à juste titre qu'elles contribuent par leurs recherches à son bien-être, son niveau de vie, sa santé, sa durabilité. Elle aspire à un monde open source, où l'information est accessible à tous, où l'interactivité et la collaboration de tous avec tous sont devenues les mots-clés d'une nouvelle langue. Jusqu'à présent, ces changements n'ont pas été pris en compte par la grande majorité de la communauté scientifique.

En tant que scientifiques, nous devons nous adapter à ces transformations. Comme le disent les Américains, " if you are not part of the solution, then you are part of the prob$\mathrm{lem}^{2}$ ». C'est à la communauté scientifique de modifier son attitude, sa manière de voir et de traiter le reste de la société, bref, d'améliorer la communication entre les STEM et le grand public en transformant ses comportements et ses institutions et en acceptant le regard critique de ses concitoyens sur ses « cuisines » scientifiques.

En tant que chercheurs, nous devons réintégrer la société, au lieu de nous retrancher derrière une apparence d'objectivité. C'est précisément parce que la communauté STEM réclame une légitimité basée sur la fiabilité de ses connaissances qu'elle doit faire clairement savoir (et de manière compréhensible pour tous) quelles en sont les limites. Nous ne pouvons pas attendre des bergers qu'ils comprennent ou communiquent les attributs de leurs épistémologies parce que leur légitimité dans la société ne relève pas de l'épistémologie.

Une réforme institutionnelle de l'organisation de la communauté des chercheurs est donc urgente, y compris une renégociation de ses rôles «scientifique » et « social ». Cette réforme devrait se fonder sur une ouverture de nos pratiques. Nous devons donner un accès complet à nos «cuisines » (nos questionnements, nos méthodes, nos données et nos théories, mais aussi comment les composantes de nos cuisines interagissent pour conduire à des résultats) ; et cela non seulement entre différentes disciplines, mais également vis-à-vis du monde extérieur.

Cela veut aussi dire admettre notre subjectivité, notre faillibilité, et nos besoins d'aide. L'attitude de « l'expert » est défensive : «Je suis l'expert; je suis le meilleur; je vous présente la vérité ». Le monde d'aujourd'hui tolère de moins en moins cette attitude. Dans l'industrie, on parle désormais $\mathrm{d}^{\prime}$ « innovation 2.0 », c'est-à-dire d'une organisation de l'innovation qui, pour accélérer le processus, partage les connaissances entre laboratoires et entreprises. La transdisciplinarité et l'ouverture de la pratique scientifique ne sont plus un choix : elles sont devenues une nécessité, sous peine de voir disparaître la communauté des STEM.

\footnotetext{
2 «Si vous ne faites pas partie de la solution, alors vous faites partie du problème ».
}

Cela mènera logiquement à refondre et revaloriser les carrières scientifiques, à donner plus d'importance à l'évaluation des équipes qu'à celle des chercheurs individuels, à redéfinir les rôles dans ces équipes, à davantage mobiliser la transdisciplinarité, et aussi et surtout à accroître l'importance de l'évaluation des bénéfices (pas seulement matériels) pour la société.

Certains visionnaires commencent à en tirer les conséquences. Une transformation de la conception et de la structure même de l'Université est en cours à Arizona State University, depuis 2002. Son président, Michael Crow, a lancé une refonte institutionnelle complète, basée sur (1) l'importance fondamentale de la fusion intellectuelle entre disciplines et la création de nouveaux terrains de recherche et d'enseignement, (2) l'engagement de 1'Université et de ses recherches dans sa communauté locale, afin de la transformer et de résoudre ses problèmes, (3) le principe d'une Université au service des étudiants plutôt que des enseignants, permettant au plus grand nombre d'accéder au plus haut niveau d'excellence, en les aidant financièrement si besoin, (4) l'idée d'une Université qui mobilise l'énergie créatrice et l'innovation de ses membres et de ses étudiants en mettant en place des structures qui favorisent la prise de risques et enfin (5) le devoir de l'Université d'avoir un rayonnement intellectuel international (Crow et Dabars, $\left.2015^{3}\right)$.

Mais tout cela est insuffisant. Il est indispensable d'œuvrer à une reconnaissance de la contextualité de la science (Wynne, 1993), et de revoir nos pratiques et institutions scientifiques en prenant en compte les changements profonds auxquels la société civile a été soumise dans les dernières décennies, tout en espérant que cette réflexion mène également à un examen critique de nos suppositions de base, pré-analytiques, constituant les cadres de nos visions et connaissances scientifiques. Dans ce processus, il faudrait connecter plus efficacement les dynamiques des communautés scientifiques et non scientifiques afin de mieux prendre en compte le contexte social des constructions scientifiques. Pour stimuler ces transitions, nous avons à l'heure actuelle surtout besoin d'intermédiaires qui réussissent à gagner la confiance de plusieurs communautés, et de leaders intellectuels qui nous offrent une réflexion pertinente sur la façon de mettre en œuvre cette transformation devenue inévitable.

\section{Références}

Atlan H., 1992. Self-organizing networks: weak, strong and intentional: the role of their under-determination, La Nuova Critica N.S., 19-20, 51-70.

\footnotetext{
3 Pour plus d'informations, consulter le site internet www.asu.edu.
} 
Churchman C.W., 1967. Wicked problems, Management Science, 14, 4, B141-B142.

Crow M.M., Dabars W.B., 2015. Designing the new American University, Baltimore: Johns Hopkins University Press.

Kahnemann D., Tversky, A., 1979. Intuitive prediction: biases and corrective procedures, Management Science, 12, 313-327.

Read D.W., van der Leeuw S.E., 2009. Biology is only part of the story..., Philosophical Transactions of the Royal Society, Series B, 363, 1959-1968.

Strumsky D., Lobo J., van der Leeuw S.E., 2011. Measuring the relative importance of reusing, recombining and creating technologies in the process of invention, Working Paper \#
11-02-003 of the Santa Fe Institute, http: / / www.santafe.edu / media/workingpapers/11-02-003.pdf.

van der Leeuw S.E., 2010. The archaeology of innovation: lessons for our times, in Innovation: perspectives for the 21st Century, Madrid, BBVA, 33-53.

van der Leeuw S.E., 2011.

Wynne B., 1993. Public uptake of science: a case for institutional reflexivity, Public Understanding of Science, 2, 321-337.

Wynne B., 1996. May the sheep safely graze? in Lash, S.M., Szerszynski B., Wynne B. (Eds), Risk, environment and modernity: towards a new ecology, London, Sage Publishers. 